\title{
Humeral Head Replacement With Wrapping Reconstruction of the Rotator Cuff After Resection of Chondrosarcoma With Long-Term Shoulder Function: A Case Report
}

\author{
Masatoshi Kamikawa ${ }^{\mathrm{a}}$, Noboru Matsumura ${ }^{\mathrm{a}, \mathrm{c}}$, Kikuzo Okada ${ }^{\mathrm{b}}$, Taku Suzuki ${ }^{\mathrm{a}}$, Robert Nakayama ${ }^{\mathrm{a}}$, \\ Takuji Iwamoto $^{a}$, Kazuki Sato ${ }^{\text {a }}$, Masaya Nakamura ${ }^{a}$, Morio Matsumoto ${ }^{a}$
}

\begin{abstract}
A 30-year-old woman with chondrosarcoma of the left proximal humerus was treated by humeral head replacement with wrapping reconstruction of the rotator cuff after resection. The dissected tendons of the rotator cuff were each sutured to the stump of the long head of the biceps brachii tendon. At the final follow-up 41 years after surgery, the patient had no difficulty in daily living and no pain, and her shoulder range of motion was successfully maintained. Although the proximal humerus is a common location for bone tumors, reconstruction and stabilization of the shoulder after tumor resection are still challenging. Wrapping reconstruction of the rotator cuff appeared to provide a dynamic force couple of the shoulder for more than 40 years. This reconstructive method is a viable treatment option for young patients with low-grade malignant tumors occurring in the proximal humerus.
\end{abstract}

Keywords: Humeral head replacement; Chondrosarcoma; Proximal humerus; Tumor reconstruction; Megaprosthesis

\section{Introduction}

Reconstruction and stabilization of the shoulder after wide resection of the proximal humerus are clinically challenging [1, 2]. Humeral head replacement is a viable option for patients with an extensive bone defect due to malignant tumor, but postoperative shoulder function is reported to be limited [1, 3]. Here, a case of humeral head replacement with wrapping reconstruction of the rotator cuff after resection of chondrosarcoma in the proximal humerus whose shoulder function was preserved for more than 40 years after surgery is reported.

Manuscript submitted January 6, 2019, accepted January 18, 2019

${ }^{\text {aDepartment }}$ of Orthopedic Surgery, Keio University School of Medicine, Tokyo, Japan

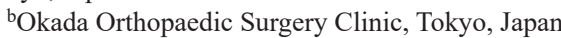

${ }^{\mathrm{c} C}$ Corresponding Author: Noboru Matsumura, Department of Orthopedic Surgery, Keio University School of Medicine, 35 Shinanomachi, Shinjuku-ku, Tokyo 160-8582, Japan. Email: noboru18@gmail.com

doi: https://doi.org/10.14740/jmc3252
The patient was informed that data concerning the case would be submitted for possible publication, and she provided consent.

\section{Case Report}

A 30-year-old woman developed left shoulder pain during golf practice in 1976, and she visited a nearby hospital. A bone tumor was seen in the left proximal humerus on plain radiographs, and she was referred to our hospital 4 days after onset. Physical examination showed slight pain and fatigability during activities in her left shoulder. However, there was no swelling or tenderness, and her shoulder range of motion was the same as that of the contralateral shoulder. Plain radiographs showed multicellular bone destruction accompanied by calcification at the epiphysis of the proximal humerus with thinning of the bone cortex (Fig. 1). The differential diagnosis of this tumor was chondrosarcoma or giant cell tumor of the bone, and surgical treatment was planned.

Surgery included resection of the proximal humerus, humeral head replacement, and reconstruction of the rotator cuff. During the resection of $6.5 \mathrm{~cm}$ of the proximal humerus through an anterior approach between the deltoid and pectoralis major, all of the rotator cuff tendons were peeled from their insertions on the tuberosities, and the long head of the biceps brachii tendon was cut at its insertion on the superior glenoid labrum. The latissimus dorsi, teres major, and pectoralis major muscles were resected from the humerus, but the deltoid muscle and axillary nerve were preserved.

The humeral head was replaced with a custom-made megaprosthesis whose articular component and stem were made from 316L stainless steel and whose metaphysis was of high-density polyethylene (Fig. 2). The stem was fixed using cement with the humeral shaft having $30^{\circ}$ of retroversion (Fig. 3 ). After replacement, the dissected tendons of the subscapularis, supraspinatus, infraspinatus, and teres minor muscles were each sutured to the stump of the long head of the biceps brachii tendon (Fig. 4). Thus, the rotator cuff did not connect with the prosthesis but wrapped the proximal part of the component by anchoring to the long head of the biceps brachii tendon.

Pathological findings included heterotypic chondrocytes proliferating in a leaf shape with hyaline cartilage matrix. Nuclear atypia was not clear, and it was diagnosed as grade 1 


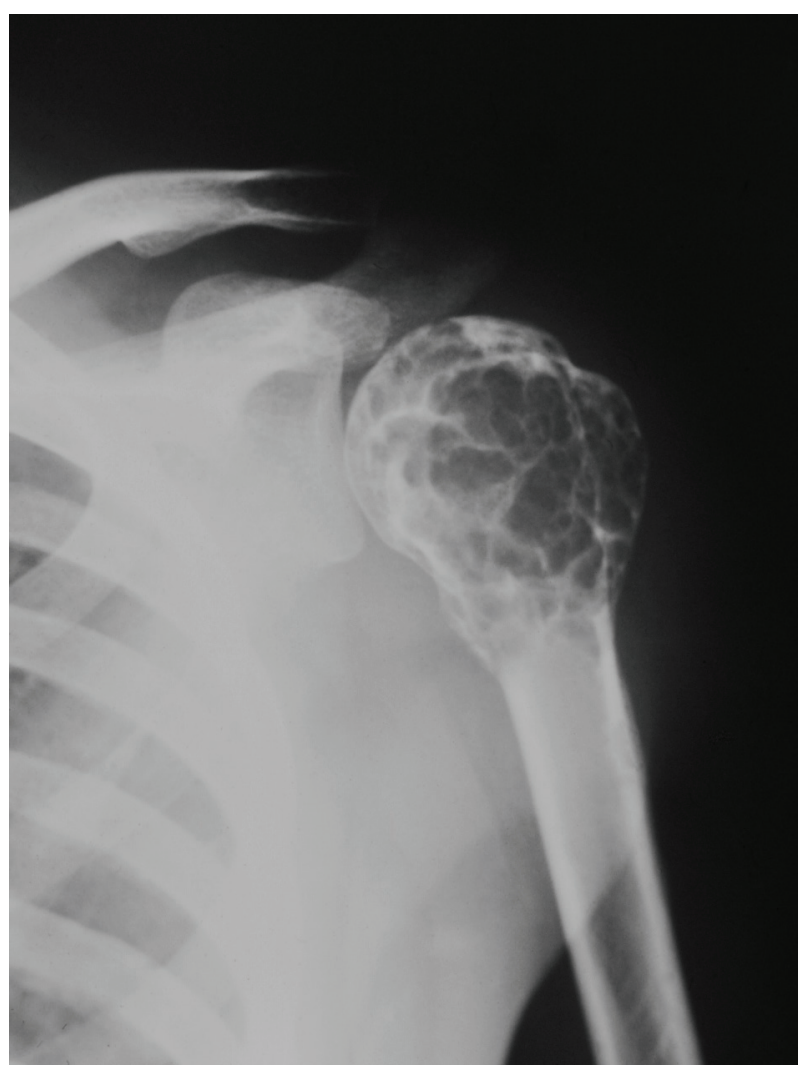

Figure 1. Preoperative radiograph showing multicellular bone destruction in the proximal humerus.

chondrosarcoma. Postoperative radiation and chemotherapy were not performed. The left arm was immobilized at $90^{\circ}$ of abduction using a plaster cast for 4 weeks after surgery. Then, passive and active shoulder motions were started.

Active shoulder motion recovered to the same level as the contralateral side 1 year after surgery (Fig. 5a), and she did not feel pain during daily activities. Shoulder range of motion was maintained during the follow-up period (Fig. 5b). At the final follow-up 41 years after surgery, active shoulder range of motion was $140^{\circ}$ in elevation, $150^{\circ}$ in abduction, $60^{\circ}$ in external rotation at $0^{\circ}$ of abduction, and at 3rd thoracic vertebral level in internal rotation behind the back (Fig. 6a-c). These ranges were slightly less than those of the contralateral shoulder, and abduction strength measured with a dynamometer was $1 \mathrm{~kg}$, but she had no difficulty in daily living and no pain. The American Shoulder and Elbow Surgeons Shoulder Score [4] was 91.6 points, and the Constant-Murley Score [5] was 73 points. Plain radiographs showed superior migration of the humeral head component and erosion on the superior glenoid cavity, but recurrence of tumor, breakage of the implant, and loosening of the stem were not found (Fig. 7).

\section{Discussion}

Although the proximal humerus is a common location for bone tumors $[1,2,6]$, reconstruction and stabilization of the shoul-

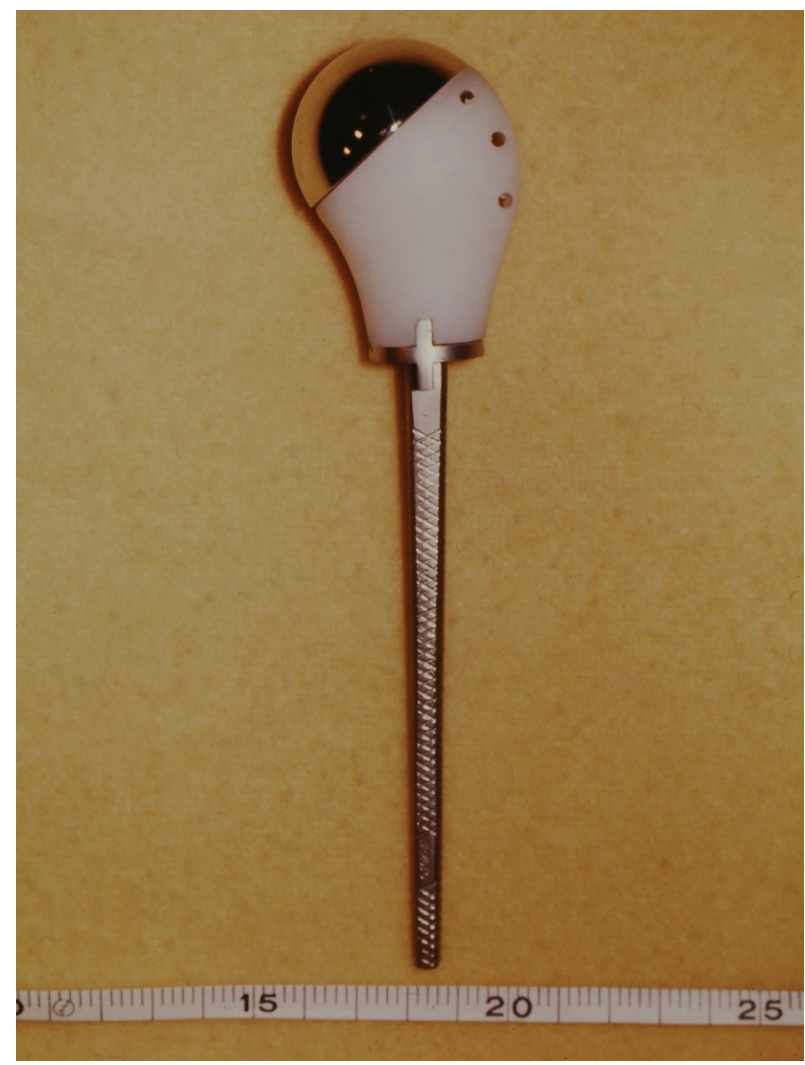

Figure 2. The custom-made megaprosthesis made from 316 L stainless steel high-density polyethylene.

der after tumor resection are still challenging $[1,2]$. Humeral head replacement with wrapping reconstruction of the rotator cuff could provide a painless shoulder with good shoulder function in a case of low-grade chondrosarcoma in the proximal humerus. The patient's shoulder range of motion was successfully maintained for more than 40 years.

The shoulder is a mobile joint with the widest range of motion in the human body, and the goal of reconstruction is to restore shoulder function. Although arthrodesis [7, 8], bone graft spacers [9], and shoulder arthroplasty [3] have been used as treatment options for extensive bone defects in the proximal humerus, these reconstructions were reported to yield limited shoulder function [1]. Humeral head replacement is a simple option, which can maintain the function of the shoulder joint with relatively low invasiveness. However, shoulder joint dysfunction is likely to occur $[8,10]$, and Fuhrmann et al [3] reported that only $50 \%$ of patients were able to elevate the arm more than $30^{\circ}$. Since shoulder function after humeral head replacement is dependent on the integrity of the rotator cuff [11], lack of the lesser and greater tuberosities, to which the rotator cuff tendons originally attach, is a serious problem with humeral head replacement for the treatment of extensive bone loss in the proximal humerus. Reverse shoulder arthroplasty [12], which was not available at the time of surgery of this patient, has become a viable alternative in recent years. However, a 30-year-old woman might be too young for this non-anatomical prosthesis even if it were available. 


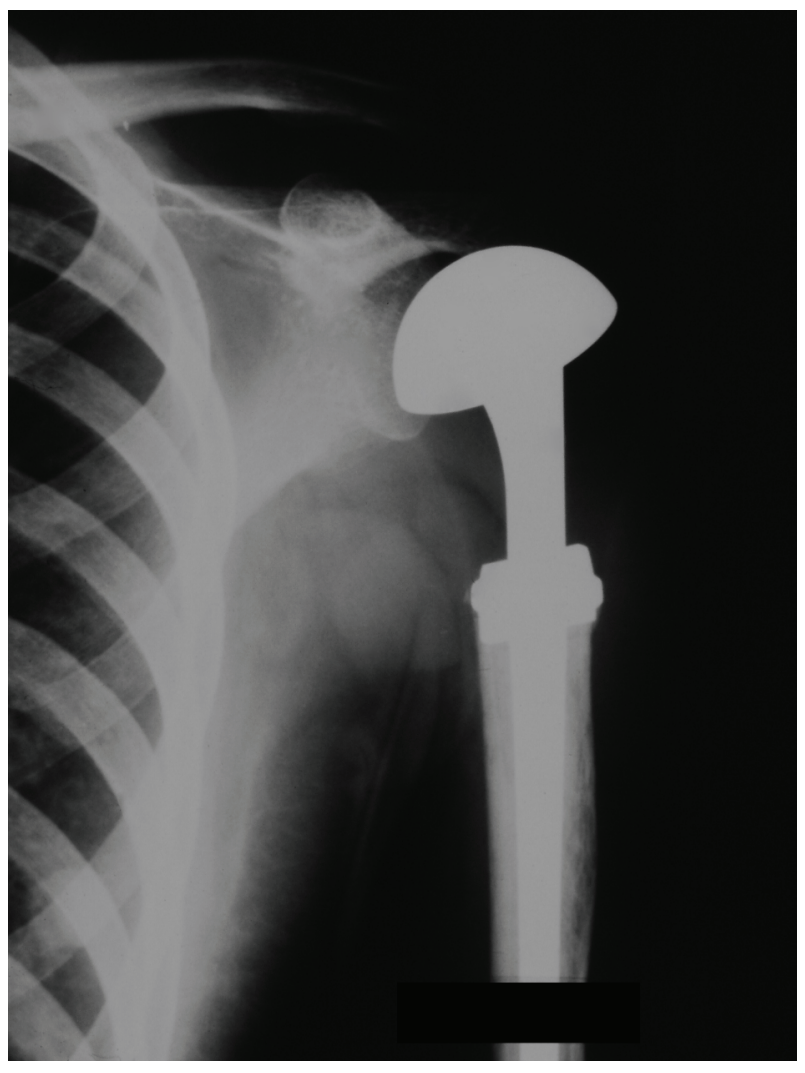

Figure 3. Postoperative radiograph. The implant is fixed to the humeral shaft using cement.

The rotator cuff tendons were dissected from their insertions, and the lesser and greater tuberosities had to be resected. In the present case, the detached rotator cuff tendons were not

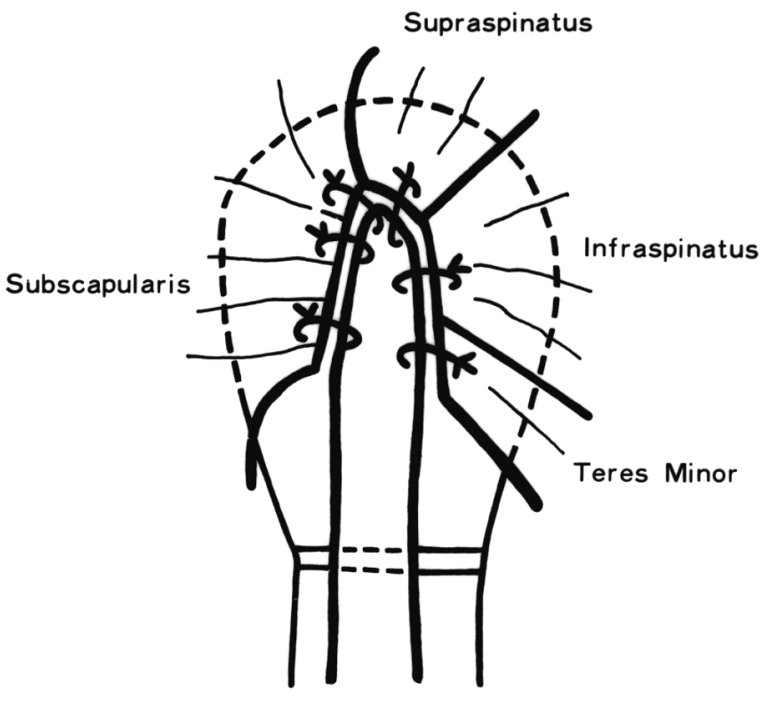

Biceps long head

Figure 4. The schematic diagram of wrapping reconstruction of the rotator cuff. The detached rotator cuff tendons are each sutured to the stump of the long head of the biceps brachii tendon.

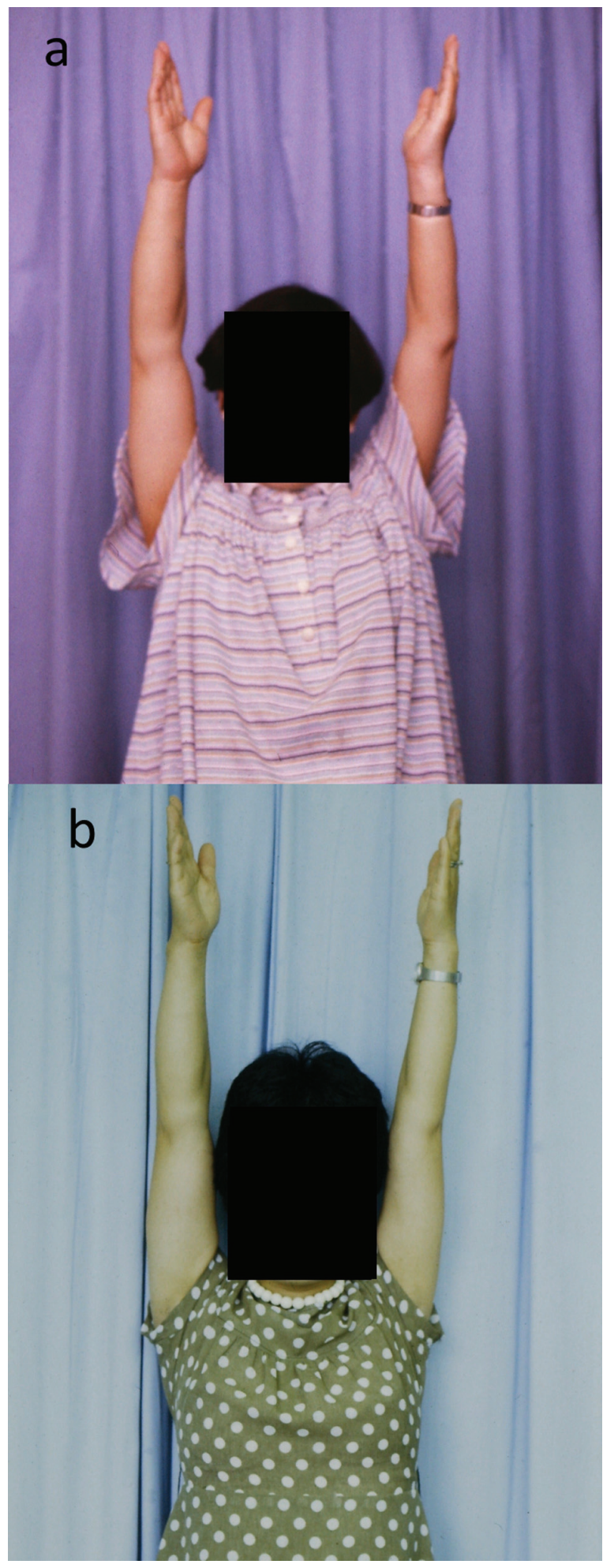

Figure 5. Shoulder function recovery after reconstruction. (a) 1 year after surgery, and (b) 5 years after surgery.

attached to the megaprosthesis but were anchored to the long head of the biceps brachii tendon. Thus, multiple different muscles were connected via the long head of the biceps brachii 


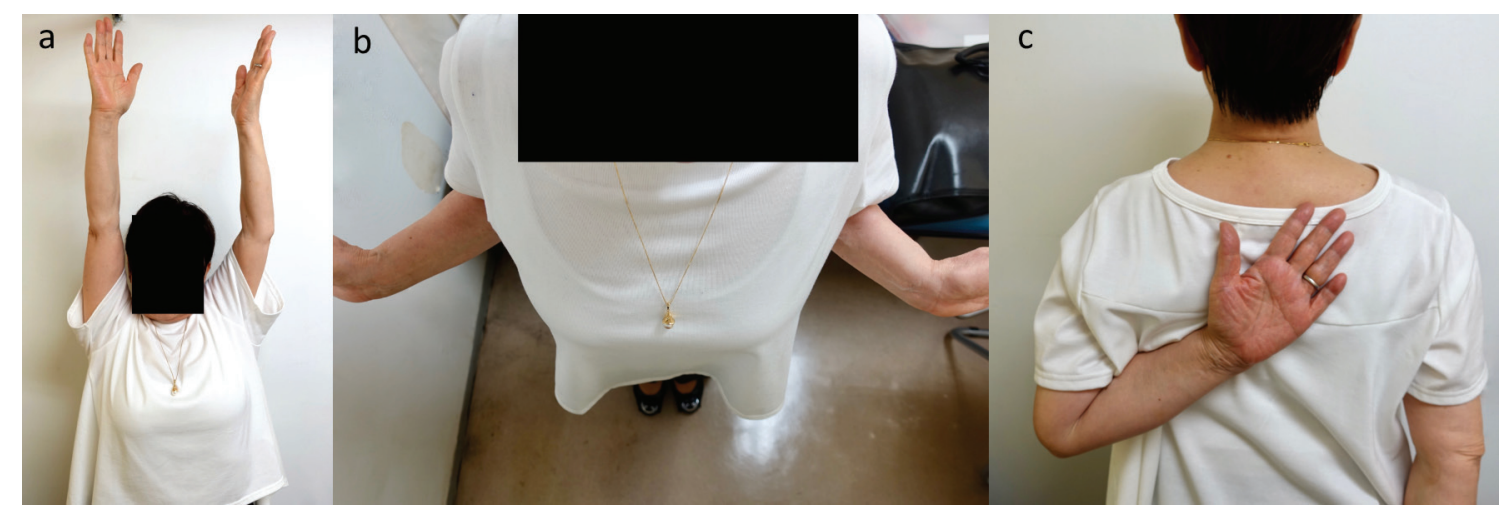

Figure 6. At the final follow-up 41 years after surgery, active shoulder range of motion is maintained. (a) $150^{\circ}$ of active elevation. (b) $60^{\circ}$ of active external rotation at $0^{\circ}$ of abduction. (c) Third thoracic vertebral level of active internal rotation behind the back.

tendon, and the custom-made megaprosthesis was wrapped by the reconstructed rotator cuff. Fortunately, the shoulder joint was successfully functional for more than 40 years, and wrapping reconstruction of the rotator cuff is thought to provide a dynamic force couple of the shoulder. Active shoulder rotation appears to be generated through the long head of the biceps brachii tendon. Although radiographs at the final follow-up showed superior migration of the humeral head component and superior glenoid erosion, which indicated rupture of a portion of the rotator cuff, we suspect that the vertical force cou-

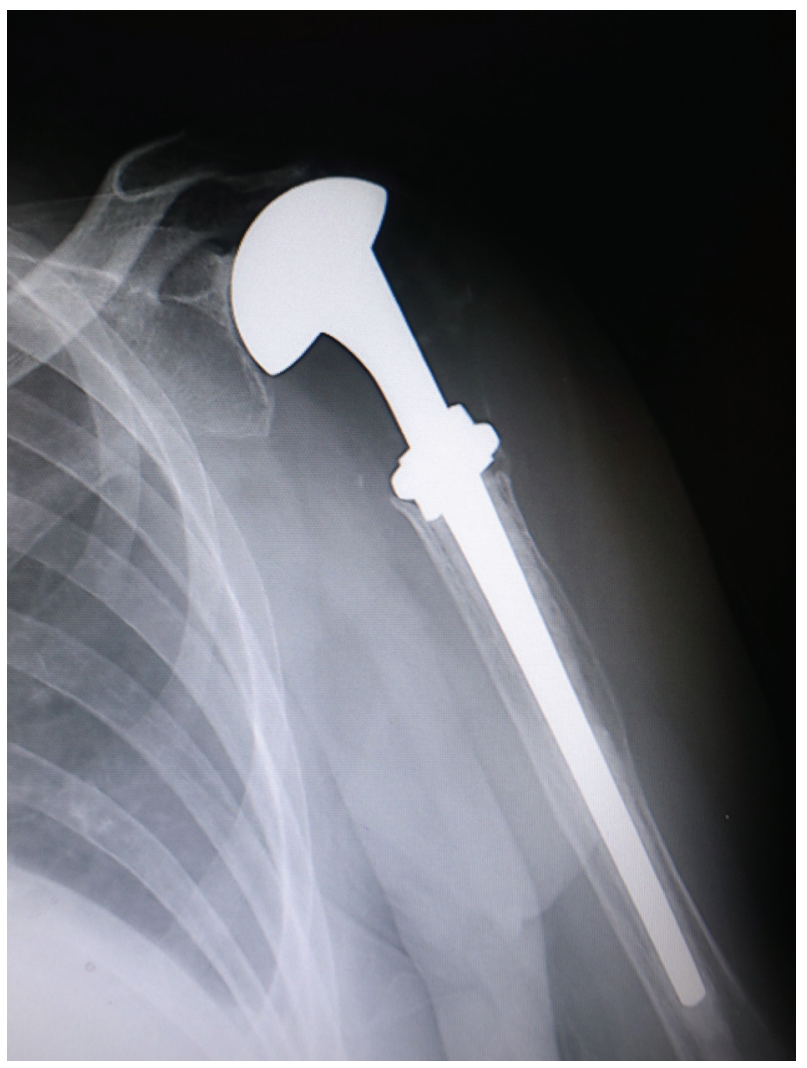

Figure 7. Plain radiograph at the time of final follow-up showing superior migration of the humeral head component and the superior glenoid erosion without stem loosening. ple of the rotator cuff was still maintained.

In this case with low-grade chondrosarcoma, the axillary nerve and deltoid muscle could be preserved. Furthermore, the quality of the preserved muscles is expected to be good because the patient was a young woman at the time of surgical intervention. Thus, the rotator cuff could be reconstructed by anchoring the muscles to the long head of the biceps brachii tendon. Humeral head replacement with wrapping reconstruction of the rotator cuff appears to be a viable treatment option for young patients with low-grade malignant tumor occurring in the proximal humerus.

\section{Acknowledgments}

We would like to thank Dr. K. Okada, who performed the operation in 1976, and named this operative technique Okada procedure.

\section{Conflict of Interest}

There are no financial or any other relationships that might lead to a conflict of interest.

\section{References}

1. Dubina A, Shiu B, Gilotra M, Hasan SA, Lerman D, Ng VY. What is the optimal reconstruction option after the resection of proximal humeral tumors? A systematic review. Open Orthop J. 2017;11:203-211.

2. Teunis T, Nota SP, Hornicek FJ, Schwab JH, Lozano-Calderon SA. Outcome after reconstruction of the proximal humerus for tumor resection: a systematic review. Clin Orthop Relat Res. 2014;472(7):2245-2253.

3. Fuhrmann RA, Roth A, Venbrocks RA. Salvage of the upper extremity in cases of tumorous destruction of the proximal humerus. J Cancer Res Clin Oncol. 2000;126(6):337-344.

4. Richards RR, An KN, Bigliani LU, Friedman RJ, Gartsman GM, Gristina AG, Iannotti JP, et al. A standardized 
method for the assessment of shoulder function. J Shoulder Elbow Surg. 1994;3(6):347-352.

5. Constant CR, Murley AH. A clinical method of functional assessment of the shoulder. Clin Orthop Relat Res. 1987;214:160-164.

6. Creighton JJ, Jr., Peimer CA, Mindell ER, Boone DC, Karakousis CP, Douglass HO. Primary malignant tumors of the upper extremity: retrospective analysis of one hundred twenty-six cases. J Hand Surg Am. 1985;10(6 Pt 1):805-814.

7. Mimata Y, Nishida J, Sato K, Suzuki Y, Doita M. Glenohumeral arthrodesis for malignant tumor of the shoulder girdle. J Shoulder Elbow Surg. 2015;24(2):174-178.

8. O'Connor MI, Sim FH, Chao EY. Limb salvage for neoplasms of the shoulder girdle. Intermediate reconstructive and functional results. J Bone Joint Surg Am. 1996;78(12):1872-1888.
9. Wada T, Usui M, Isu K, Yamawakii S, Ishii S. Reconstruction and limb salvage after resection for malignant bone tumour of the proximal humerus. A sling procedure using a free vascularised fibular graft. J Bone Joint Surg Br. 1999;81(5):808-813.

10. Ross AC, Wilson JN, Scales JT. Endoprosthetic replacement of the proximal humerus. J Bone Joint Surg Br. 1987;69(4):656-661.

11. Kontakis G, Koutras C, Tosounidis T, Giannoudis P. Early management of proximal humeral fractures with hemiarthroplasty: a systematic review. J Bone Joint Surg Br. 2008;90(11):1407-1413.

12. King JJ, Nystrom LM, Reimer NB, Gibbs CP, Jr., Scarborough MT, Wright TW. Allograft-prosthetic composite reverse total shoulder arthroplasty for reconstruction of proximal humerus tumor resections. J Shoulder Elbow Surg. 2016;25(1):45-54. 